\title{
Comtesse de Sabran-Chevalier de Boufflers, Correspondance (1777-1785 Le lit bleu et 1786-1787 La Promesse)
}

\section{Elena Aschieri}

\section{(2) OpenEdition \\ Journals}

\section{Edizione digitale}

URL: http://journals.openedition.org/studifrancesi/5966

DOI: 10.4000/studifrancesi.5966

ISSN: 2421-5856

\section{Editore}

Rosenberg \& Sellier

\section{Edizione cartacea}

Data di pubblicazione: 1 mai 2011

Paginazione: 175-176

ISSN: 0039-2944

Notizia bibliografica digitale

Elena Aschieri, «Comtesse de Sabran-Chevalier de Boufflers, Correspondance (1777-1785 Le lit bleu et 1786-1787 La Promesse)», Studi Francesi [Online], 163 (LV | I) | 2011, online dal 30 novembre 2015, consultato il 12 janvier 2021. URL: http://journals.openedition.org/studifrancesi/5966 ; DOI: https:// doi.org/10.4000/studifrancesi.5966

Questo documento è stato generato automaticamente il 12 janvier 2021.

\section{cc) (†)}

Studi Francesi è distribuita con Licenza Creative Commons Attribuzione - Non commerciale - Non opere derivate 4.0 Internazionale. 


\title{
Comtesse de Sabran-Chevalier de Boufflers, Correspondance (1777-1785 Le lit bleu et 1786-1787 La Promesse)
}

\author{
Elena Aschieri
}

\section{NOTIZIA}

Comtesse de Sabran Chevalier de Boufflers, Correspondance (1777-1785 Le lit bleu et 1786-1787 La Promesse). Edition établie et présentée par Sue CARRELL, Paris, Tallendier, 2009 et 2010, pp. 366 et 588.

1 Presentiamo i primi due volumi - almeno un altro sarà prossimamente pubblicato -, della ricca e fitta corrispondenza tra la Comtesse de Sabran e il Chevalier de Boufflers, frutto di un minuzioso lavoro di archivio e ricomposizione del testo ad opera di Sue Carrell, specialista di letteratura epistolare francese del Settecento. Se infatti parte di questa raccolta di lettere era già stata pubblicata nel 1875 e poi nel 1891, solo con questa nuova edizione - ricostruita sui manoscritti, di cui buona parte inediti -, abbiamo un'idea fedele dell'opera in questione. Opera poiché, come buona parte delle epistole (amorose) del xvIII secolo che nascevano come private, venivano rese pubbliche, ad un certo punto, almeno da uno dei due mittenti, così che noi ci troviamo di fronte ad un doppio giornale che si legge spesso come un romanzo.

2 Tema fondamentale è un amore felice che si sviluppa nell'arco di moltissimi anni (dieci in questi due volumi, tutta una vita, come sappiamo e potremo vedere nei due prossimi tomi). Nel 1777 il Prince de Ligne presenta al Chevalier de Boufflers, celibe, Mme de Sabran, vedova. Nonostante la loro situazione, le convenances, gli interessi di fortuna e di famiglia, non autorizzano il matrimonio, che avrà luogo solo dopo la Rivoluzione, nel 1797. Il carattere corretto e ligio di Mme de Sabran, la cui reputazione non fu mai messa in dubbio, fa iniziare la corrispondenza sotto il segno di un'amicizia fraterna, ma si percepisce fin dall'inizio che i protagonisti lottano contro una forza invincibile: così gli 
appellativi «ma sœur», «mon frère», lasceranno spazio a «ma femme», «mon mari», più insistenti dopo il maggio 1781, quando i due passeranno la loro prima notte insieme nel Lit bleu della contessa. Il primo dei due volumi viene così intitolato dalla curatrice, il secondo, La Promesse, fa riferimento, invece, alla promessa reciproca dei due amanti di scriversi quotidianamente, dopo che nel 1785 Boufflers è nominato governatore del Senegal, status che gli consente di ottenere una situazione economica e sociale degna della Contessa.

3 Il Lit bleu ci presenta i primi otto anni dello scambio amoureux: è l'amore, infatti, l'argomento centrale, la sua evoluzione, un vero percorso delle sue diverse tappe in cui la corte paziente e appassionata del Chevalier supera molte prove. Assistiamo poi, una volta conquistata la Contessa, agli alti e bassi della passione amorosa condita da dichiarazioni appassionate, gelosie, riconciliazioni, compromessi. Pochi sono i riferimenti a situazioni pubbliche o a considerazioni private che invece accompagnano il discorso amoroso del secondo volume. Scopriamo così un mondo ozioso e noioso di nobili legati alla corte reale, a Marie Antoinette, in cui mille aneddoti a carattere mondano ma anche sociale, letterario, artistico, filosofico e politico (dalla guerra contro l'Inghilterra, all'affaire $d u$ collier, alle tensioni prerivoluzionarie tra il Re e i parlamentari) vengono a movimentare la monotonia della vita quotidiana della Contessa, lontana dal Chevalier e dedita soprattutto ai suoi due adorati figli, Delphine e Elzéar.

4 Le lettere della Contessa, in cui l'analisi della propria disperazione tocca a volte il "patetico", contengono elementi utili alla ricostruzione della vita dei nobili francesi intorno alla Corte, sono vivaci, ricche di esprit e di sensibilité, tipici del Tournant des Lumières. Boufflers apprezzava le doti di scrittrice di Mme de Sabran e il suo stile: «je défierais Mme de Sévigné, Mme de Lambert et Mme du Deffand réunies d'entrer en lice». Le lettere del Chevalier, condite da massime morali, potrebbero essere considerate quasi lettere di viaggio: ci permettono di scoprire l'Africa del xVIII secolo, «la terre fertile d'un rouge foncé du Cap vert, les drogues les plus rares, les conditions de vie, les paysages grandioses et ce peuple qu'il commence à connaître et à respecter» come fa osservare la curatrice, lasciandoci anche una forte testimonianza contro la tratta dei neri («ces mots de captifs, de captives, d'esclaves, de chaînes, de fer etc., me font toujours saigner le cœur»). Ci segnalano, infine, le forti preoccupazioni per le malattie (tema presentissimo anche nelle lettere della Contessa) e soprattutto per la sofferenza del corpo esposto alle alte temperature, la diffidenza verso i medici; esse sono inoltre ricche di riferimenti letterari e di meditazioni filosofiche sull'esistenza umana («la navigation est d'une lenteur à faire rendre l'âme», una navigazione che è spesso immagine della vita: «que la vie est longue et qu'elle est courte! C'est une vaste mer, tantôt calme, tantôt irritée, toujours perfide, semée de mille écueils, et par-ci parlà d'îles délicieuses»).

5 Riconosciamo le difficoltà dell'impresa di Sue Carrell. L'edizione, esito di una ricerca continua iniziata nel 1986, è ineccepibile. Le lettere sono state raccolte così come i due protagonisti probabilmente le ricevettero (ricostruzione più semplice laddove la data consentiva l'ordine logico, ma anche complessa, che ha richiesto una ricerca più approfondita, per le lettere non datate). La curatrice ha raggruppato le lettere sotto il comune denominatore che le contraddistingue, scandendo in questo modo le tappe della straordinaria histoire d'amour. 
6 Ricchissime e varie nel contenuto le note: presentano i personaggi dell'epoca nominati, chiariscono spesso passaggi poco chiari o completano il quadro di una situazione con una visione più ampia dell'argomento trattato (da una questione politica, al gioco di carte del pharaon, dalla nascita e poi dalla morte della prima figlia di Maria Antonietta agli avvenimenti di Corte), riportando spesso frammenti di altre corrispondenze dell'epoca, rinviano a opere e citazioni di autori più o meno noti, scovando anche le imprecisioni dei due mittenti e delle precedenti edizioni. Il primo volume, Le lit bleu, contiene l'«Avant propos» (pp. 11-17), la «Note sur l'édition 1777-1785» (pp. 19-21), che illustra brevemente la «Constitution du texte» e i criteri adottati per questa edizione, e l'«Introduction» (pp. 23-40) che, partendo dalla biografia dei due amanti, traccia il quadro sociale e storico in cui essi vivono. In fondo al volume, dopo le lettere degli anni 1777-1785, nelle «Annexes» (pp. 345-366), viene riprodotto la novella Aline, reine de Golconde, che diede a Boufflers la gloria letteraria, la Lettre du chevalier de Boufflers à l'abbé Parquet, e alcuni suoi Poèmes.

7 Il secondo volume, La Promesse, contiene, oltre alle lettere degli anni 1786-1787, la «Note sur l'édition 1786-1787» (pp.11-14) ricca, come abbiamo detto, di inediti, alcuni dei quali acquistati e posseduti ora dalla Carrell, altri di proprietà degli eredi della famiglia Sabran. Attendiamo con ansia i prossimi volumi che completeranno questo eccellente lavoro. 